\title{
Lights and Shadows in the Genetics of Syndromic and Non-Syndromic Hearing Loss in the Italian Population
}

\author{
Anna Morgan ${ }^{1, *}$, Stefania Lenarduzzi ${ }^{1}$, Beatrice Spedicati ${ }^{1,2}{ }^{\circledR}$, Elisabetta Cattaruzzi ${ }^{1}$, \\ Flora Maria Murru ${ }^{1}$, Giulia Pelliccione ${ }^{1}$, Daniela Mazzà ${ }^{1}$, Marcella Zollino ${ }^{3,4}$ (D), \\ Claudio Graziano ${ }^{5}$ D, Umberto Ambrosetti ${ }^{6}$, Marco Seri ${ }^{5}$, Flavio Faletra ${ }^{1}$ (D) \\ and Giorgia Girotto ${ }^{1,2}$ \\ 1 Institute for Maternal and Child Health-IRCCS “Burlo Garofolo", 34137 Trieste, Italy; \\ stefania.lenarduzzi@burlo.trieste.it (S.L.); beatrice.spedicati@burlo.trieste.it (B.S.); \\ elisabetta.cattaruzzi@burlo.trieste.it (E.C.); floramaria.murru@burlo.trieste.it (F.M.M.); \\ giulia.pelliccione@burlo.trieste.it (G.P.); daniela.mazza@burlo.trieste.it (D.M.); \\ flavio.faletra@burlo.trieste.it (F.F.); giorgia.girotto@burlo.trieste.it (G.G.) \\ 2 Department of Medicine, Surgery and Health Sciences, University of Trieste, 34125 Trieste, Italy \\ 3 Fondazione Policlinico Universitario A. Gemelli, IRCCS, UOC Genetica, 00168 Rome, Italy; \\ Marcella.Zollino@Unicatt.it \\ 4 Istituto di Medicina Genomica, Università Cattolica Sacro Cuore, 00168 Rome, Italy \\ 5 Unit of Medical Genetics, S. Orsola-Malpighi Hospital, 40138 Bologna, Italy; \\ claudio.graziano@unibo.it (C.G.); marco.seri@unibo.it (M.S.) \\ 6 Audiology and audiophonology, University of Milano/Fondazione IRCCS Cà Granda Ospedale Maggiore \\ Policlinico, 20122 Milano, Italy; umberto.ambrosetti@unimi.it \\ * Correspondence: anna.morgan@burlo.trieste.it
}

Received: 1 October 2020; Accepted: 20 October 2020; Published: 22 October 2020

check for updates

\begin{abstract}
Hearing loss (HL), both syndromic (SHL) and non-syndromic (NSHL), is the most common sensory disorder, affecting 460 million people worldwide. More than $50 \%$ of the congenital/childhood cases are attributable to genetic causes, highlighting the importance of genetic testing in this class of disorders. Here we applied a multi-step strategy for the molecular diagnosis of HL in 125 patients, which included: (1) an accurate clinical evaluation, (2) the analysis of GJB2, GJB6, and MT-RNR1 genes, (3) the evaluation STRC-CATSPER2 and OTOA deletions via Multiplex Ligation Probe Amplification (MLPA), (4) Whole Exome Sequencing (WES) in patients negative to steps 2 and 3. Our approach led to the characterization of $50 \%$ of the NSHL cases, confirming both the relevant role of the GJB2 $(20 \%$ of cases) and STRC deletions (6\% of cases), and the high genetic heterogeneity of NSHL. Moreover, due to the genetic findings, $4 \%$ of apparent NSHL patients have been re-diagnosed as SHL. Finally, WES characterized $86 \%$ of SHL patients, supporting the role of already know disease-genes. Overall, our approach proved to be efficient in identifying the molecular cause of HL, providing essential information for the patients' future management.
\end{abstract}

Keywords: hereditary hearing loss; MLPA; whole exome sequencing; molecular diagnosis

\section{Introduction}

Hereditary Hearing Loss (HHL) is the most common sensory disorder in childhood and adulthood, affecting approximately $1-3$ out of 1000 newborns [1].

Genetic factors account for more than $50 \%$ of all the cases, where the majority exhibit an autosomal recessive (AR) pattern of inheritance (75-80\%) followed by $20-25 \%$ of autosomal dominant (AD) cases and $1-1.5 \%$ of $\mathrm{X}$-linked or mitochondrial ones [2]. 
More than 200 genes (i.e., $~ 1 \%$ of all the coding genes) are involved in the hearing process [3]; therefore, it is not surprising that HHL displays substantial genetic heterogeneity. The many genetic forms of hearing loss can be further categorized into syndromic and non-syndromic conditions, which, respectively, constitute $30 \%$ and $70 \%$ of the genetic causes of congenital HHL, with syndromic HHL likely underestimated [4,5]. To date, about 170 loci and 117 genes (36 autosomal dominant (AD), 65 autosomal recessives (AR), $11 \mathrm{AD} / \mathrm{AR}$, and $5 \mathrm{X}$-linked genes) have been reported as causative of Non-Syndromic Hearing Loss (NSHL) (Hereditary Hearing Loss Homepage; http://hereditaryhearingloss.org/), and more than 400 syndromes associated with hearing loss and other symptoms (Syndromic Hearing Loss-SHL) have been described [6]. In particular, among the syndromes identified so far, some of them appear more frequently than the others (e.g., Usher syndrome compared to Waardenburg syndrome) $[7,8])$, although, in some cases, the full spectrum of clinical features might be subtle, or even not present until later in life [9].

The implementation of next-generation sequencing technologies (NGS), together with molecular karyotyping (e.g., Single Nucleotide Polymorphism (SNP) array or Comparative Genomic Hybridization (CGH) array) and other validating assays (e.g., Multiplex Ligation Probe Amplification-MLPA), has dramatically increased the diagnostic rate of HHL, leading to the identification of several mutations and Copy Number Variations (CNVs) in known deafness genes, as well as to the discovery of new disease genes [10-13]. The possibility to simultaneously screen large number of genes is essential to address with the genetic heterogeneity of HHL. This aspect is fundamental for NSHL, where, apart from the relevant contribution of the GJB2 gene and, in some populations of GJB6 gene, both responsible for $\sim 50 \%$ of all AR cases [14-16], and of STRC deletions (accounting for $1 \%$ to $5 \%$ of HL cases [17]), no other worldwide primary players have been identified.

In the present work, we applied a multi-step strategy to identify the genetic cause of HHL in a subset of 125 individuals recruited in the last two years. The protocol included: (1) an accurate clinical evaluation of all the patients and their relatives to exclude all the cases in which HL was due to non-genetic causes (i.e., middle ear anomalies, infections, ototoxic drugs, etc.) and to distinguish between NSHL and SHL; (2) the analysis of GJB2, GJB6 and MT-RNR1 genes in patients affected by NSHL; (3) the evaluation of STRC-CATSPER2 and OTOA deletions in case of negativity to step (2); (4) Whole Exome Sequencing (WES) analysis in case of negativity to steps (2) and (3) and for patients affected by SHL (Figure 1).

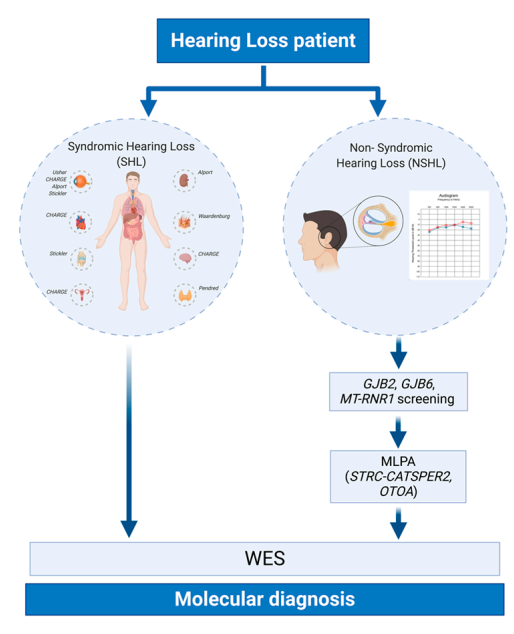

Figure 1. Schematic representation of the multi-step strategy applied for the study of Hereditary Hearing Loss (HHL). All of the patients enrolled in the present study underwent a careful clinical examination to distinguish between Syndromic Hearing Loss (SHL) and Non-Syndromic Hearing Loss (NSHL). Afterwards, NSHL patients were screened for mutation in GJB2, GJB6, and MT-RNR1 genes, and for deletions in STRC-CATSPER2 and OTOA genes. All of the NSHL patients negative to the first-level screening, together with SHL patients, have been then analyzed through Whole Exome Sequencing (WES). 
The present work results illustrate the genetic heterogeneity of HHL and the importance of a detailed clinical characterization combined with high-throughput technologies for the diagnosis of both NSHL and SHL.

\section{Materials and Methods}

\subsection{Ethical Statement}

All patients signed written informed consent forms for both genetic counseling and molecular genetic testing. In the case of minors, informed consent was obtained from the next of kin. The study was approved by the Institutional Review Board (IRB) of the Institute for Maternal and Child Health (IRCCS) Burlo Garofolo, Trieste, Italy. All research was conducted according to the ethical standards as defined by the Helsinki Declaration.

\subsection{Clinical Evaluation and Sample Collection}

A total of 125 HHL patients have been recruited in the following centers (Otorhinolaryngology or Medical Genetics): Trieste (IRCCS Burlo Garofolo), Milano (IRCCS Cà Granda-Ospedale Maggiore Policlinico), Rome (Policlinico Universitario "A. Gemelli"), and Bologna (Policlinico S. Orsola-Malpighi).

All participants underwent pure tone audiometric testing (PTA) or auditory brainstem response (ABR) in order to characterize the severity of HL according to the international guidelines described by Clark (1981) [18]. Moreover, neurological and ophthalmological examinations, electrocardiogram, kidney ultrasonography, Magnetic Resonance Imaging (MRI) and Computerized Tomography (CT) scan, and thyroid function were carried out on routine basis in all probands.

Based on the clinical findings, 118 patients were classified as NSHL and seven as SHL. Furthermore, depending on the pedigree structure, the cases were divided into sporadic $(n=90)$ and familial $(n=35)$, the latter being classified as likely autosomal recessive (AR) $(n=9)$, and likely autosomal dominant (AD) $(n=26)$.

\subsection{GJB2, GJB6, and mtDNA Analysis}

For all the patients, the entire coding region of GJB2 was analyzed by Sanger sequencing (primers available upon request). According to the manufacturer's instructions, DNA was sequenced on a $3500 \mathrm{Dx}$ Genetic Analyzer (Life-Technologies, Carlsbad, CA, USA), using ABI PRISM 3.1 Big Dye terminator chemistry (Life Technologies, Carlsbad, CA, USA). Moreover, GJB6 deletions (D13S1830-D13S1854) were screened by multiplex PCR using the method described by del Castillo et al. 2002 [19], while The A1555G mtDNA mutation was tested by Restriction Fragment Length Polymorphism (PCR-RFLP) analysis using BsmAI as restriction enzyme, followed by visualization on an agarose gel stained with ethidium bromide.

\subsection{Multiplex Ligation Probe Amplification (MLPA)}

MLPA analysis for identification of deletion/duplication in STRC-CATSPER2 and OTOA genes was conducted using SALSA ${ }^{\circledR}$ MLPA $^{\circledR}$ probe mixes P461-A1 DIS (MRC-Holland, Amsterdam, the Netherlands) according to the manufacturer's instructions. In brief, 50-100 ng DNA was denatured and hybridized overnight at $60{ }^{\circ} \mathrm{C}$ with the SALSA ${ }^{\circledR}$ probe mix. Samples were then treated with DNA ligase for $15 \mathrm{~min}$ at $54{ }^{\circ} \mathrm{C}$. The reaction was stopped by incubation at $98{ }^{\circ} \mathrm{C}$ for $5 \mathrm{~min}$. Finally, PCR amplification was carried out with specific fluorescent-labeled PCR primers. After amplification, the amplified products' fragment analysis was performed on ABI 3500dx Genetic Analyzer (Life-Technologies, Carlsbad, CA, USA).

Coffalyser.Net software was employed for data analysis in combination with the lot-specific MLPA Coffalyser sheet. The dosage quotient (DQ) of the reference probes in the patient samples was between 0.80 and 1.20. The following cutoff values for the DQ of the probes were used to interpret MLPA results; $0.80<\mathrm{DQ}<1.20$ (no deletion/duplication), $\mathrm{DQ}=0$ (deletion), and $1.75<\mathrm{DQ}<2.15$ (duplication). 


\subsection{Whole Exome Sequencing (WES)}

WES was completed on an Illumina NextSeq 550 instrument (Illumina, San Diego, CA, USA) with NextEra Flex for enrichment-Exome panel reagents, according to the manufacturer's protocol.

Secondary analysis has been carried out using Isis Software (v.2.5.42.5-Illumina, Illumina, San Diego, CA, USA), i.e., reads alignment with Burrows-Wheeler Aligner (BWA) 0.7.7-isis-1.0.2 and variant calling with Isaac Variant Caller v. 2.1.4.2.

Single Nucleotides Variations (SNVs) and small Insertions and Deletions (INDELs) were collected into a standardized Variant Call Format (VCF) version 4.1 [20]. SNVs and INDELS were then annotated with ANNOVAR [21] using human genome build 19 (hg19) as the reference.

SNVs leading to synonymous amino acids substitutions not predicted as damaging, not affecting splicing or highly conserved residues were excluded, as well as SNVs/INDELs with quality score (QUAL) $<20$ and called in off-target regions.

A comparison between the identified genetic variants and data reported in NCBI dbSNP build153 (http://www.ncbi.nlm.nih.gov/SNP/) as well as in gnomAD (http://gnomad.broadinstitute.org/), and National Heart, Lung and Blood Institute (NHLBI) Exome Sequencing Project (ESP) Exome Variant Server (Exome Variant Server, NHLBI GO Exome Sequencing Project (ESP), Seattle, WA) led to the exclusion of those variants previously reported as polymorphism. In particular, a Minor Allele Frequency (MAF) cutoff of 0.005 for recessive forms and 0.001 for the dominant ones was used.

The pathogenicity of known genetic variants was evaluated using ClinVar (http://www.ncbi. nlm.nih.gov/clinvar/), Deafness Variation Database (http://deafnessvariationdatabase.org/), and The Human Gene Mutation Database (http://www.hgmd.cf.ac.uk/ac/index.php).

Several in silico tools, such as PolyPhen-2 [22], Sorting Intolerant from Toleran (SIFT) [23], MutationTaster [24], Likelihood Ratio Test (LRT) [25], and Combined Annotation Dependent Depletion (CADD) score [26] were used to evaluate the pathogenicity of novel variants. Moreover, the evolutionary conservation of residues across species was evaluated by phyloP [27] and Genomic Evolutionary Rate Profiling (GERP) [28] scores.

Human Splicing Finder (HSF) version 2.4.1 [29] and Splice Site Prediction by Neural Network (NNSPLICE) version 9 (www.fruitfly.org) were adopted to predict the effect of the splice site mutations.

Finally, on a patient by patient basis, variants were discussed in the context of phenotypic data at interdisciplinary meetings and the most likely disease-causing SNVs/INDELs were analyzed by direct Sanger sequencing.

Sanger sequencing was also employed to perform the segregation analysis within the Family.

\section{Results}

During the last two years, 125 patients have been tested for GJB2, GJB6, and the A1555G mitochondrial mutation. Twenty percent of them were positive for mutations in the GJB2 gene (i.e., 25 patients) with the c.35delG being the most frequent mutation (i.e., $44 \%$ of patients c. $35 \mathrm{delG}$ homozygotes and $48 \%$ c.35delG carriers, together with other in-trans mutations, such as the p.(Glu120del), p.(Trp24*) and p.(Glu47*)) (Table 1). None of them carried deletions in GJB6 or the A1555G mutation in the mitochondrial gene MT-RNR1.

Ninety-three NSHL individuals negative to this first-level screening were analyzed through MLPA to search for STRC-CATSPER 2 and OTOA deletions. Overall $8 \%$ of cases carried a homozygous deletion in STRC $(n=6)$ or OTOA $(n=1)$ genes (Table 2$)$. The remaining 86 patients, together with their relatives, were then analyzed using WES. Sequencing data analysis led to the molecular diagnosis in 26 additional patients (Table 2) reaching an overall detection rate (i.e., GJB2/GJB6/mtDNA screening, MLPA, WES) of 50\% (Figure 2A,B). 
Table 1. List of patients positive for GJB2 gene with indication of the identified mutations.

\begin{tabular}{|c|c|c|c|c|}
\hline Patient ID & Gene & Variant 1 & Variant 2 & Status \\
\hline Patient 1 & & c.35delG, p.(Gly12Valfs * 2) & c. $-27 \mathrm{C}>\mathrm{T}$ & compound heterozygous \\
\hline Patient 2 & & c.35delG, p.(Gly12Valfs * 2) & c.229T > C, p.(Trp77Arg) & compound heterozygous \\
\hline Patient 3 & & c.35delG, p.(Gly12Valfs * 2) & c.35delG, p.(Gly12Valfs * 2) & homozygous \\
\hline Patient 4 & & c.35delG, p.(Gly12Valfs * 2) & c.139G > T p. $\left(\right.$ Glu $\left.47^{*}\right)$ & compound heterozygous \\
\hline Patient 5 & & c.35delG, p.(Gly12Valfs * 2) & c.358_360delGAG, p.(Glu120del) & compound heterozygous \\
\hline Patient 6 & & c.35delG, p.(Gly12Valfs * 2) & c. $-23+1 \mathrm{G}>\mathrm{A}$ & compound heterozygous \\
\hline Patient 7 & & c.35delG, p.(Gly12Valfs * 2) & c.71G > A, p. $($ Glu24 *) & compound heterozygous \\
\hline Patient 8 & & c.35delG, p.(Gly12Valfs * 2) & c.71G > A, p. $($ Glu24 *) & compound heterozygous \\
\hline Patient 9 & & c.35delG, p.(Gly12Valfs * 2) & c.35delG, p.(Gly12Valfs * 2) & homozygous \\
\hline Patient 10 & & c.35delG, p.(Gly12Valfs * 2) & c.35delG, p.(Gly12Valfs * 2) & homozygous \\
\hline Patient 11 & & c.35delG, p.(Gly12Valfs * 2) & c.314_327del14, p.(Lys105Argfs * 5) & compound heterozygous \\
\hline Patient 12 & & c.35delG, p. $($ Gly12Valfs * 2) & c.35delG, p.(Gly12Valfs * 2) & homozygous \\
\hline Patient 13 & GJB2 & c.35delG, p.(Gly12Valfs * 2) & c.95G > A, p.(Arg32His) & compound heterozygous \\
\hline Patient 14 & & c.35delG, p.(Gly12Valfs * 2) & c.35delG, p.(Gly12Valfs * 2) & homozygous \\
\hline Patient 15 & & c.59T > C, p.(Ile20Thr) & c.314_327del14, p.(Lys105Argfs * 5) & compound heterozygous \\
\hline Patient 16 & & c.35delG, p. $($ Gly12Valfs * 2) & c.71G > A, p. $($ Glu24 * ) & compound heterozygous \\
\hline Patient 17 & & c.35delG, p.(Gly12Valfs* 2) & c.35delG, p.(Gly12Valfs * 2) & homozygous \\
\hline Patient 18 & & c.35delG, p.(Gly12Valfs * 2) & c.139G > T p. $\left(\right.$ Glu $\left.47^{*}\right)$ & compound heterozygous \\
\hline Patient 19 & & c.35delG, p.(Gly12Valfs * 2) & c.35delG, p.(Gly12Valfs * 2) & homozygous \\
\hline Patient 20 & & c. 35 delG, p. $($ Gly12Valfs * $)$ & c.35delG, p.(Gly12Valfs * 2) & homozygous \\
\hline Patient 21 & & c.101T > C, p.(Met34Thr) & c.358_360delGAG, p.(Glu120del) & compound heterozygous \\
\hline Patient 22 & & c. 35 delG, p. $($ Gly12Valfs * $)$ & c.35delG, p.(Gly12Valfs * 2) & homozygous \\
\hline Patient 23 & & c.35delG, p.(Gly12Valfs * 2) & c.35delG, p.(Gly12Valfs * 2) & homozygous \\
\hline Patient 24 & & c.35delG, p.(Gly12Valfs * 2) & c.358_360delGAG, p.(Glu120del) & compound heterozygous \\
\hline Patient 25 & & c.35delG, p. $($ Gly12Valfs * 2) & c.35delG, p.(Gly12Valfs * 2) & homozygous \\
\hline
\end{tabular}


Table 2. List of likely causative variants identified by WES and Multiplex Ligation Probe Amplification (MLPA). All variants have been classified based on their frequency reported in Genome Aggregation Database (gnomAD, http://gnomad.broadinstitute.org/) and their pathogenicity. In particular, the following tools have been used: CADD PHRED (Pathogenicity score: > 10 predicted to be deleterious), GERP++_RS (higher number is more conserved), and Polyphen-2 (D: Probably damaging, P: possibly damaging; B: benign), SIFT (D: deleterious; T: tolerated), MutationTaster (A (disease_causing_automatic); D (disease_causing); N (polymorphism); $\mathrm{P}$ (polymorphism automatic)). NA = not available; hom = homozygous; het = heterozygous.

\begin{tabular}{|c|c|c|c|c|c|c|c|c|c|c|c|}
\hline Family ID & Gene & cDNA Change & Protein Change & dbSNP & gnomAD_ALL & $\begin{array}{l}\text { CADD } \\
\text { PHRED }\end{array}$ & $\begin{array}{c}\text { GERP } \\
+++R S\end{array}$ & Polyphen-2 & SIFT & $\begin{array}{c}\text { Mutation } \\
\text { Taster }\end{array}$ & References \\
\hline Family 3 & $\begin{array}{c}\text { OTOA } \\
\text { (NM_144672.3) }\end{array}$ & $\begin{array}{l}\text { entire gene deletion } \\
\text { (hom) }\end{array}$ & NA & NA & NA & NA & NA & NA & NA & NA & $\begin{array}{l}\text { Shearer et al., (2014) } \\
\text { Genome Med [30] }\end{array}$ \\
\hline Family 4 & $\begin{array}{c}\text { COL11A2 } \\
(\mathrm{NM}-080680.2)\end{array}$ & c. $3100 \mathrm{C}>\mathrm{T}$ (het) & p.(Arg1034Cys) & rs121912947 & NA & 32 & 2.95 & D & $\mathrm{D}$ & $\mathrm{D}$ & $\begin{array}{l}\text { McGuirt et al., (1999) } \\
\text { Nat Genet [31] }\end{array}$ \\
\hline Patient 41 & $\begin{array}{c}M Y O 7 A \\
\left(N M \_000260.3\right)\end{array}$ & c.6236G > A (hom) & p.(Arg2079Gln) & rs765083332 & 0.00004188 & 25.5 & 4.24 & $\mathrm{P}$ & $\mathrm{T}$ & D & NA \\
\hline \multirow[b]{2}{*}{ Patient 42} & & c.10084C > T (het) & \multirow{2}{*}{$\begin{array}{c}\text { p.(Gln3362*) } \\
\text { p.(Asn4553Glufs * } \\
\text { 18) }\end{array}$} & NA & NA & 42 & 3.02 & NA & NA & A & NA \\
\hline & (NM_032119.3) & c.13655dupT (het) & & rs765376986 & NA & NA & NA & NA & NA & NA & NA \\
\hline Patient 44 & $\begin{array}{c}\text { STRC } \\
\left(\mathrm{NM} \_153700.2\right)\end{array}$ & $\begin{array}{l}\text { entire gene deletion } \\
\text { (hom) }\end{array}$ & NA & NA & NA & NA & NA & NA & NA & NA & $\begin{array}{c}\text { Vona et al.,(2015) Clin } \\
\text { Genet [32] }\end{array}$ \\
\hline Patient 45 & $\begin{array}{c}\text { GJB1 } \\
\left(N M_{0} 000166.5\right)\end{array}$ & c.790C > T (het) & p.(Arg264Cys) & rs587777879 & 0.00005714 & 25.8 & 4.9 & D & D & D & $\begin{array}{l}\text { Numakura et al., } \\
\text { (2002) Hum Mutat [33] }\end{array}$ \\
\hline Patient 51 & $\begin{array}{c}\text { CLDN14 } \\
\text { (NM_144492.2) }\end{array}$ & c.301G > A (hom) & p.(Gly101Arg) & rs74315438 & 0.00004302 & 26.5 & 5.42 & $\mathrm{D}$ & $\mathrm{D}$ & $\mathrm{D}$ & $\begin{array}{c}\text { Wattenhofer et } \\
\text { al.,(2005) Hum Mutat } \\
\text { [34] }\end{array}$ \\
\hline Family 6 & $\begin{array}{c}\text { SMPX } \\
\left(N M_{0} 014332.2\right)\end{array}$ & $\begin{array}{c}\text { c.45+1G > A } \\
\text { (hemizygous) }\end{array}$ & NA & NA & NA & 24.4 & 4.9 & NA & NA & D & NA \\
\hline Family 7 & $\begin{array}{c}\text { SIX1 } \\
\left(N M \_005982.3\right)\end{array}$ & $\begin{array}{l}\text { c.397_399delGAG } \\
\text { (het) }\end{array}$ & p.(Glu133del) & rs80356460 & NA & NA & NA & NA & NA & NA & $\begin{array}{c}\text { Ruf et al., (2004) Proc } \\
\text { Natl Acad Sci U S A } \\
\text { [35] }\end{array}$ \\
\hline Family 8 & $\begin{array}{c}\text { KARS } \\
\text { (NM_001130089.1) }\end{array}$ & $\begin{array}{l}\text { c. } 1423 \mathrm{C}>\mathrm{T} \text { (het) } \\
\text { c. } 1570 \mathrm{~T}>\mathrm{C} \text { (het) }\end{array}$ & $\begin{array}{l}\text { p.(Leu475Phe) } \\
\text { p.(Cys524Arg) }\end{array}$ & $\begin{array}{l}\text { NA } \\
\text { NA }\end{array}$ & $\begin{array}{l}\text { NA } \\
\text { NA }\end{array}$ & $\begin{array}{l}28.2 \\
26.9\end{array}$ & $\begin{array}{l}5.91 \\
5.81\end{array}$ & $\begin{array}{l}\text { P } \\
D\end{array}$ & $\begin{array}{l}\mathrm{T} \\
\mathrm{D}\end{array}$ & $\begin{array}{l}\text { D } \\
\text { D }\end{array}$ & $\begin{array}{l}\text { NA } \\
\text { NA }\end{array}$ \\
\hline Patient 57 & $\begin{array}{c}\text { OTOG } \\
\left(N M \_001277269.1\right)\end{array}$ & c. $2500 \mathrm{C}>\mathrm{T}$ (hom) & p. $(G \ln 834 *)$ & rs554847663 & 0.0004274 & 37 & 2.01 & NA & NA & $\mathrm{D}$ & $\begin{array}{l}\text { Sheppard et al.,(2018) } \\
\text { Genet Med [36] }\end{array}$ \\
\hline Family 10 & $\begin{array}{c}\text { PAX3 } \\
\text { (NM_181457.3) }\end{array}$ & c.220C > T (het) & p.(Arg74Cys) & NA & NA & 35 & 5.24 & $\mathrm{D}$ & $\mathrm{D}$ & $\mathrm{D}$ & $\begin{array}{l}\text { Lenarduzzi et al., } \\
\text { (2019) Hear Res [37] }\end{array}$ \\
\hline
\end{tabular}


Table 2. Cont

\begin{tabular}{|c|c|c|c|c|c|c|c|c|c|c|c|}
\hline Family ID & Gene & cDNA Change & Protein Change & dbSNP & gnomAD_ALL & $\begin{array}{l}\text { CADD } \\
\text { PHRED } \\
\end{array}$ & $\begin{array}{c}\text { GERP } \\
+++R S\end{array}$ & Polyphen-2 & SIFT & $\begin{array}{c}\text { Mutation } \\
\text { Taster }\end{array}$ & References \\
\hline Patient 62 & $\begin{array}{c}\text { STRC } \\
\left(N M \_153700.2\right)\end{array}$ & $\begin{array}{l}\text { entire gene deletion } \\
\text { (hom) }\end{array}$ & NA & NA & NA & NA & NA & NA & NA & NA & $\begin{array}{l}\text { Vona et al.,(2015) Clin } \\
\text { Genet [32] }\end{array}$ \\
\hline Family 13 & $\begin{array}{c}E S P N \\
\left(N M \_031475.2\right)\end{array}$ & $\begin{array}{l}\text { entire gene deletion } \\
\text { (hom) }\end{array}$ & NA & NA & NA & NA & NA & NA & NA & NA & NA \\
\hline Family 14 & $\begin{array}{c}\text { MYO6 } \\
\text { (NM_004999.3) }\end{array}$ & c.1525delG (het) & p.(Val509Trpfs * 7) & NA & NA & NA & NA & NA & NA & $\mathrm{D}$ & NA \\
\hline Family 15 & $\begin{array}{c}\text { OTOF } \\
\left(N M \_194248.2\right)\end{array}$ & c. $2891 \mathrm{C}>\mathrm{A}$ (hom) & p.(Ala964Glu) & rs201329629 & NA & 32 & 5.41 & $\mathrm{D}$ & $\mathrm{D}$ & D & $\begin{array}{l}\text { Rodriguez-Ballesteros } \\
\text { et al. (2008) Hum } \\
\text { Mutat [38] }\end{array}$ \\
\hline \multirow[t]{2}{*}{ Patient 65} & \multirow[t]{2}{*}{$\begin{array}{c}\text { ADGRV1 } \\
\text { (NM_032119.3) }\end{array}$} & c. $4378 \mathrm{G}>\mathrm{A}$ (het) & p.(Gly1460Ser) & rs1303930496 & 0.00001248 & 32 & 5.53 & $\mathrm{D}$ & $\mathrm{D}$ & $\mathrm{D}$ & $\begin{array}{l}\text { Magliulo et al.,(2017) } \\
\text { Otolaryngol Head } \\
\text { Neck Surg [39] }\end{array}$ \\
\hline & & c.13655dupT (het) & $\begin{array}{l}\text { p.(Asn4553Glufs * } \\
18)\end{array}$ & rs765376986 & NA & NA & NA & NA & NA & NA & NA \\
\hline Family 18 & $\begin{array}{c}\text { DIAPH1 } \\
\text { (NM_005219.4) }\end{array}$ & c.3556delC (het) & p. (Leu1186Serfs * 2) & NA & NA & NA & NA & NA & NA & $\mathrm{D}$ & NA \\
\hline Patient 70 & $\begin{array}{c}\bar{G} A T A 3 \\
\left(N M \_002051.2\right)\end{array}$ & $\begin{array}{c}\text { c. } 925-1 \mathrm{G}>\mathrm{T} \text { (het, de } \\
\text { novo) }\end{array}$ & NA & NA & NA & 25.9 & 5.3 & NA & NA & NA & NA \\
\hline Family 20 & $\begin{array}{c}\text { TECTA } \\
\left(\mathrm{NM} \_005422.2\right)\end{array}$ & c.3841T > C (het) & p.(Cys1281Arg) & NA & NA & 17.03 & 5.76 & $\mathrm{~T}$ & $\mathrm{D}$ & $\mathrm{D}$ & NA \\
\hline Family 21 & $\begin{array}{c}\text { STRC } \\
\left(N M_{1} 153700.2\right)\end{array}$ & $\begin{array}{l}\text { entire gene deletion } \\
\text { (hom) }\end{array}$ & NA & NA & NA & NA & NA & NA & NA & NA & $\begin{array}{c}\text { Vona et al.,(2015) Clin } \\
\text { Genet [32] }\end{array}$ \\
\hline \multirow{3}{*}{ Family 23} & & c. $2276 \mathrm{G}>\mathrm{T}$ (het) & p.(Cys759Phe) & rs80338902 & 0.000947 & 33 & 5.79 & $\mathrm{D}$ & $\mathrm{D}$ & D & $\begin{array}{l}\text { Rivolta et al.,(2000) } \\
\text { Am J Hum Genet [40] }\end{array}$ \\
\hline & (NM_206933.2) & c.11864G > A (het) & p.(Trp3955*) & rs111033364 & 0.000119 & 51 & 5.53 & NA & $\mathrm{D}$ & $\mathrm{D}$ & $\begin{array}{l}\text { van Wijk et al., (2004) } \\
\text { Am J Hum Genet [41] }\end{array}$ \\
\hline & $\begin{array}{c}\text { EYA4 } \\
\left(N N_{0} 04100.4\right)\end{array}$ & c.714C > A (het) & p.(Tyr238*) & rs1264401894 & 0.000004 & 37 & 4.77 & NA & $\mathrm{D}$ & $\mathrm{D}$ & NA \\
\hline Family 24 & $\begin{array}{c}\text { WFS1 } \\
\text { (NM_006005.3) }\end{array}$ & c. $2567 \mathrm{C}>\mathrm{A}$ (het) & p.(Pro856His) & NA & NA & 23.7 & 4.82 & $\mathrm{D}$ & $\mathrm{D}$ & $\mathrm{D}$ & NA \\
\hline Family 26 & $\begin{array}{c}\text { MYO6 } \\
\text { (NM_004999.3) }\end{array}$ & c. $613 \mathrm{C}>\mathrm{T}$ (het) & p. $(\operatorname{Arg} 205 *)$ & rs557441143 & 0.00000398 & 37 & 3.31 & NA & NA & A & $\begin{array}{l}\text { Choi ET AL.,(2013) } \\
\text { PLoS One } 8 \text { [42] }\end{array}$ \\
\hline Family 27 & $\begin{array}{c}\text { COL2A1 } \\
\left(N M \_001844.5\right)\end{array}$ & c. $4201 \mathrm{G}>\mathrm{C}$ (het) & p.(Asp1401His) & NA & NA & 19.9 & 5.06 & $\mathrm{D}$ & $\mathrm{D}$ & $\mathrm{D}$ & NA \\
\hline Family 28 & $\begin{array}{c}\text { ATP2B2 } \\
\text { (NM_001001331.4) }\end{array}$ & c. $962 C>$ G (het) & p.(Ser321*) & NA & NA & 42 & 5.34 & NA & $\mathrm{T}$ & A & NA \\
\hline
\end{tabular}


Table 2. Cont.

\begin{tabular}{|c|c|c|c|c|c|c|c|c|c|c|c|}
\hline Family ID & Gene & cDNA Change & Protein Change & dbSNP & gnomAD_ALL & $\begin{array}{l}\text { CADD } \\
\text { PHRED }\end{array}$ & $\begin{array}{r}\text { GERP } \\
+++R S\end{array}$ & Polyphen-2 & SIFT & $\begin{array}{c}\text { Mutation } \\
\text { Taster }\end{array}$ & References \\
\hline Patient 79 & LARS2 & $\begin{array}{l}4 \mathrm{~Kb} \text { gene deletion } \\
\text { (hom) }\end{array}$ & NA & NA & NA & NA & NA & NA & NA & NA & NA \\
\hline \multirow{2}{*}{ Family 29} & \multirow{2}{*}{$\begin{array}{c}\text { PDZD7 } \\
(\mathrm{NM} 024895.4)\end{array}$} & c.166dupC (het) & p. $($ Arg56Profs * 24) & rs587776894 & NA & NA & NA & NA & NA & NA & $\begin{array}{l}\text { Ebermann et al.,(2010) } \\
\text { J Clin Invest [43] }\end{array}$ \\
\hline & & c.305G > A (het) & p.(Arg102His) & rs760825921 & 0.00001061 & 34 & 5.07 & $\mathrm{D}$ & $\mathrm{D}$ & $\mathrm{D}$ & NA \\
\hline Family 30 & $\begin{array}{c}\text { STRC } \\
\left(\mathrm{NM} \_153700.2\right)\end{array}$ & $\begin{array}{l}\text { entire gene deletion } \\
\text { (hom) }\end{array}$ & NA & NA & NA & NA & NA & NA & NA & NA & $\begin{array}{l}\text { Vona et al.,(2015) Clin } \\
\text { Genet [32] }\end{array}$ \\
\hline Family 31 & $\begin{array}{c}\text { STRC } \\
\left(\mathrm{NM} \_153700.2\right)\end{array}$ & $\begin{array}{l}\text { entire gene deletion } \\
\text { (hom) }\end{array}$ & NA & NA & NA & NA & NA & NA & NA & NA & $\begin{array}{c}\text { Vona et al.,(2015) Clin } \\
\text { Genet [32] }\end{array}$ \\
\hline Family 32 & $\begin{array}{c}\text { HOMER2 } \\
\left(N M \_199330.2\right)\end{array}$ & $\begin{array}{l}\text { c.592_597delACCACA } \\
\text { (het) }\end{array}$ & p.(Thr198_Thr199del) & NA & NA & NA & NA & NA & NA & $\mathrm{P}$ & NA \\
\hline Patient 84 & $\begin{array}{c}\text { STRC } \\
\left(N M_{1} 153700.2\right)\end{array}$ & $\begin{array}{l}\text { entire gene deletion } \\
\text { (hom) }\end{array}$ & NA & NA & NA & NA & NA & NA & NA & NA & $\begin{array}{c}\text { Vona et al.,(2015) Clin } \\
\text { Genet [32] }\end{array}$ \\
\hline Family 34 & $\begin{array}{c}\text { STRC } \\
\left(N M \_153700.2\right)\end{array}$ & c.4057C > T (hom) & p. $(G \ln 1353 *)$ & rs774312182 & 0.00006374 & 37 & 4.16 & NA & NA & $\mathrm{D}$ & $\begin{array}{c}\text { Shearer et al., (2010) } \\
\text { Proc Natl Acad Sci U S } \\
\text { A [44] }\end{array}$ \\
\hline Patient 86 & $\begin{array}{c}\text { TCOF1 } \\
\text { (NM_001135243.1) }\end{array}$ & c.4362_4366del (het) & $\begin{array}{l}\text { p.(Lys1457Glufs * } \\
\text { 11) }\end{array}$ & NA & NA & NA & NA & NA & NA & NA & NA \\
\hline Family 35 & $\begin{array}{c}\text { COL4A3 } \\
\left(N M \_000091.4\right)\end{array}$ & c. $3943 \mathrm{C}>\mathrm{T}$ (het) & p.(Pro1315Ser) & rs760703010 & 0.00002793 & 25 & 5.57 & $\mathrm{D}$ & $\mathrm{D}$ & $\mathrm{D}$ & NA \\
\hline Patient 87 & $\begin{array}{c}\text { USH2A } \\
\text { (NM_206933.2) }\end{array}$ & c.11864G > A (hom) & p. $(\operatorname{Trp} 3955 *)$ & rs111033364 & 0.000119 & 51 & 5.53 & NA & $\mathrm{D}$ & A & $\begin{array}{l}\text { van Wijk et al., (2004) } \\
\text { Am J Hum Genet [41] }\end{array}$ \\
\hline Patient 88 & $\begin{array}{c}\text { USH2A } \\
\text { (NM_206933.2) }\end{array}$ & c.4933G > T (hom) & p.(Gly1645*) & NA & NA & 38 & 3.86 & NA & NA & A & $\begin{array}{l}\text { Sloan-Heggen et } \\
\text { al.,(2016) Hum Genet } \\
\text { [45] }\end{array}$ \\
\hline \multirow[b]{2}{*}{ Patient 89} & \multirow{2}{*}{$\begin{array}{c}\text { USH2A } \\
\text { (NM_206933.2) }\end{array}$} & c.2035G > T (het) & p. $\left(\right.$ Gly $\left.679^{*}\right)$ & NA & NA & 38 & 5.26 & NA & NA & A & NA \\
\hline & & c. $11864 \mathrm{G}>\mathrm{A}$ (het) & p. $\left(\operatorname{Trp} 3955^{*}\right)$ & rs111033364 & 0.000119 & 51 & 5.53 & NA & $\mathrm{D}$ & A & $\begin{array}{l}\text { van Wijk et al., (2004) } \\
\text { Am J Hum Genet [41] }\end{array}$ \\
\hline \multirow{2}{*}{ Patient 90} & \multirow{2}{*}{$\begin{array}{c}\text { MYO7A } \\
\text { (NM_000260.3) }\end{array}$} & c.735G > A (het) & (p.Gln245Gln) & NA & NA & NA & NA & NA & NA & A & $\begin{array}{l}\text { Atik et al.,(2015) PLoS } \\
\text { One } 10 \text { [46] }\end{array}$ \\
\hline & & $\begin{array}{l}\text { c.1834_1836delAGC } \\
\text { (het) }\end{array}$ & p.(Ser612del) & NA & NA & NA & NA & NA & NA & $\mathrm{D}$ & NA \\
\hline
\end{tabular}




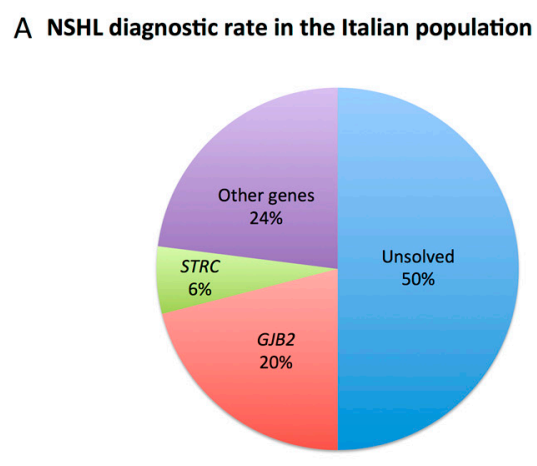

B Genes distribution in NSHL/NSHL-mimic Italian patients

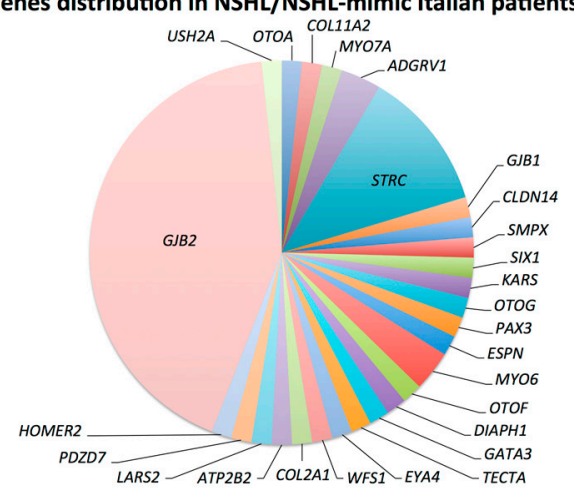

C

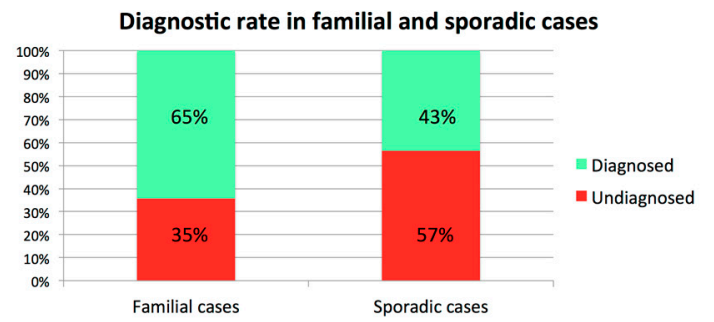

Figure 2. Diagnostic rate and genes distribution in NSHL patients. (A) Overall diagnostic rate for NSHL. Moreover, $50 \%$ of patients received a conclusive molecular diagnosis, with GJB2 being the most frequently mutated gene (i.e., 20\%), followed by STRC (i.e., 6\%). (B) Genes distribution among all the NSHL and NSHL-mimic patients investigated. (C) Diagnostic rate comparison between familial and sporadic cases showing a higher percentage of solved cases among patients presenting with a familial history of HL.

In particular, $65 \%$ of familial cases (i.e., 22/34) were genetically characterized, while for sporadic cases, the molecular cause was identified in $43 \%$ of patients (i.e., 36/84) (Figure 2C).

WES data allowed unveiling some peculiar scenarios, which reflect the complexity of NSHL.

In particular, WES allowed: (1) to detect syndromes in patients displaying only subtle phenotypic features; (2) to early diagnose diseases with a late-onset clinical manifestations; (3) to identify mutations in more than one gene involved in the same phenotype; (4) to molecularly characterize multiple genetic conditions in the same patient; and (5) to clarify the role of recently discovered genes.

An example of 1) is Family 10, who came to genetic counseling with a clinical diagnosis of NSHL in the proband and in the mother. WES revealed the presence of a novel heterozygous variant in PAX3 (NM_181457.3) (c.220C > T p.(Arg74Cys)), which occurred as de novo in the mother and was inherited from the proband. PAX3 is a gene known for being causative of Waardenburg syndrome type 1 and 2 [47], a disease characterized by HL, pigmentation abnormalities and, in some cases, dystopia canthorum or other additional features [8]. A clinical re-evaluation of the patients revealed the presence of mild pigmentary disturbances of the iris, hair, and skin, confirming the molecular diagnosis.

Regarding point 2), three patients who only displayed sensorineural hearing loss have been molecularly classified as Usher patients. In particular, two of them carried pathogenic mutations in the GPR98 gene (NM_032119.3) while one carried two compound heterozygous mutations in USH2A (NM_206933.2) gene (Table 2).

As for point number 3), we were able to identify the simultaneous presence of mutations in both USH2A and EYA4 genes in Family 23, an Italian family apparently affected by autosomal dominant NSHL. WES revealed the presence of two compound heterozygous mutations in USH2A (NM_206933.2) in the proband (i.e., c.11864G > A, p.(Trp3955*) and c.2276G > T, p.(Cys759Phe)) in addition to a stop gain variant in EYA4 (NM_004100.4), i.e., c.714C > A, p.(Tyr238*), segregating in the other affected family members (i.e., the proband's mother, the maternal uncle and the maternal grandfather). 
Subsequently, the proband's hearing thresholds appeared worse than those of her relatives, possibly due to the simultaneous presence of mutations in both USH2A and EYA4A.

In other cases, WES revealed the presence of 4) multiple independent genetic conditions that were initially misinterpreted as a single syndrome. An example is Patient 84 , who presented with sensorineural hearing loss and periventricular nodular heterotopia (Figure 3).

A

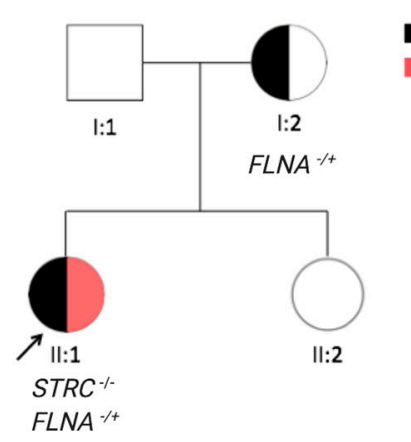

B

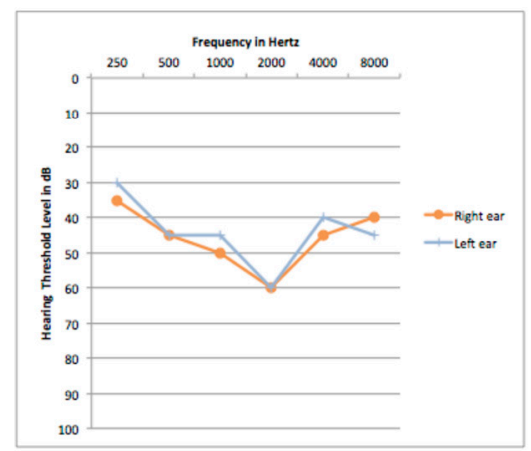

C
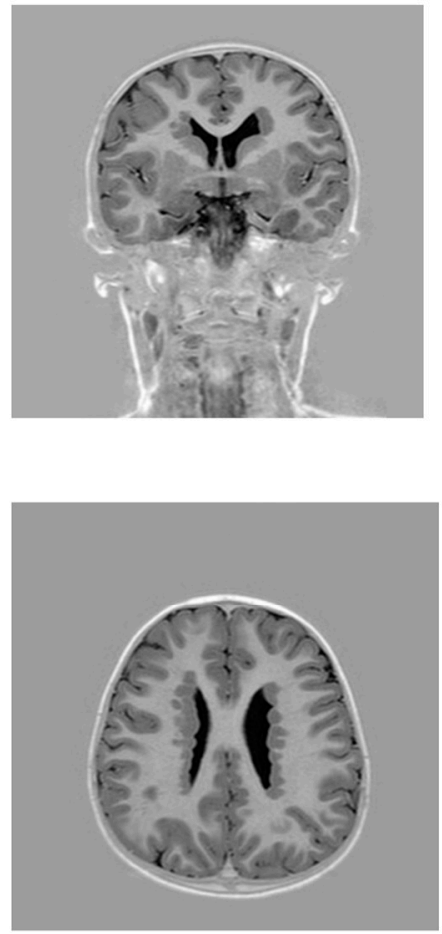

Figure 3. Pedigree, clinical and genetic features of Patient 84. (A) Pedigree of Patient 84, affected by both sensorineural hearing loss and periventricular nodular heterotopia. (B) Audiometric features of the affected individual, displayed as audiograms (air conduction). The thresholds of the right and left ears are shown. (C) Axial (coronal) scan IR T1-weighted. Bilateral periventricular nodules of grey matter are seen immediately deep to the ependymal layer of the bodies of both lateral ventricles.

MLPA detected a homozygous deletion in the STRC gene explaining the HL phenotype but not the neurological one. The application of WES allowed to identify a heterozygous nonsense variant in the FLNA gene ((NM_001456.3), c.1159C > T p. $\left.\left(\mathrm{Gln} 387^{*}\right)\right)$, a gene known for being causative of periventricular nodular heterotopia in an X-linked dominant fashion [48]. The variant was inherited from the mother, whose MRI revealed foci of periventricular nodular heterotopia, confirming the identified allele's pathogenic effect.

WES also allowed 5) to detect novel variants in genes recently described as causative of NSHL, supporting their pathogenic role. An example is the case of Family 28, an Italian family presenting with a likely autosomal dominant NSHL (Figure 4A), where a novel nonsense variant in ATP2B2 ((NM_001001331.4) c.962C > G, p.(Ser321 *) has been identified. 


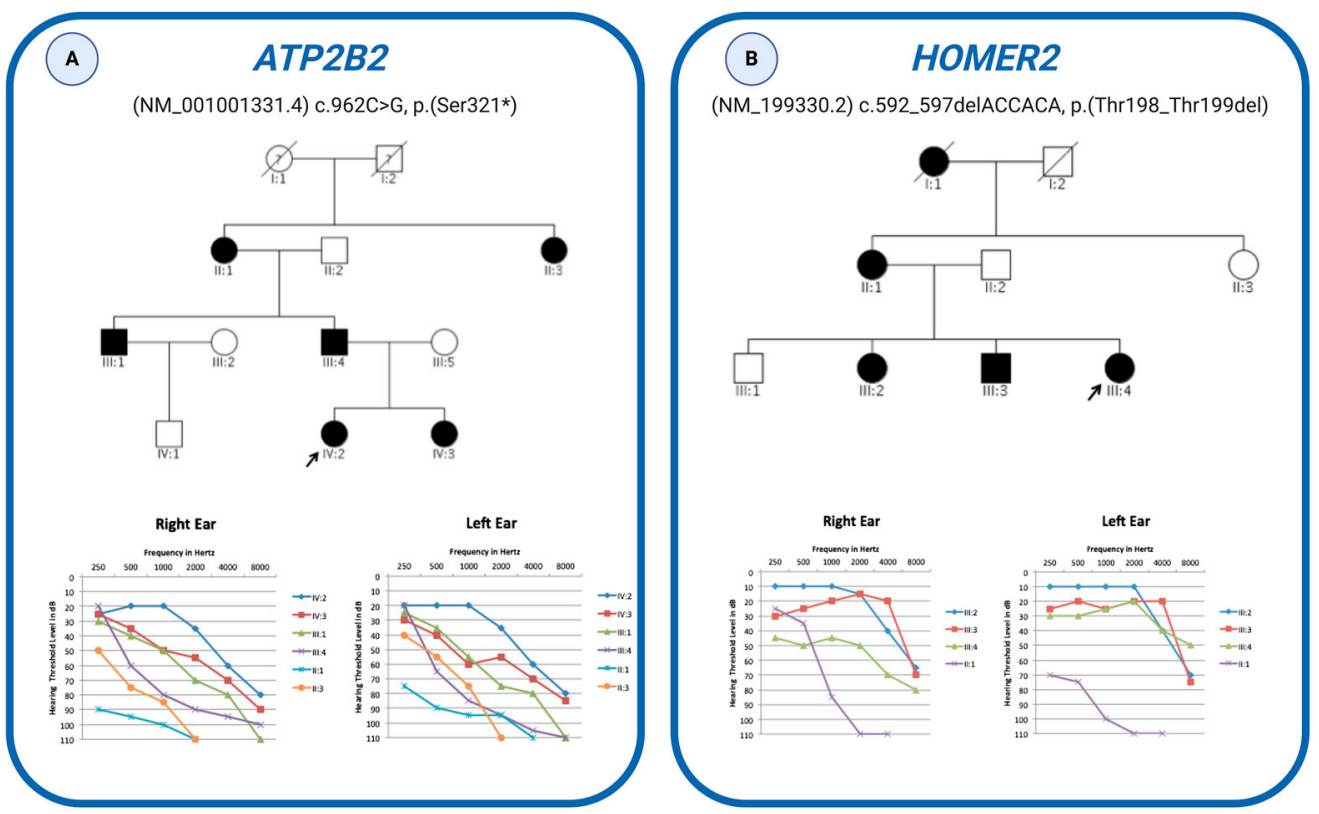

Figure 4. Pedigree and audiometric features of the families with novel variants in $A T P 2 B 2$ and HOMER2 genes. (A) Pedigree of the family carrying a novel nonsense variant in the ATP2B2 gene and audiometric features of the affected individuals. (B) Pedigree of the family carrying a novel deletion in the HOMER2 gene and audiometric features of the affected individuals. Filled symbols represent affected individuals. Probands are indicated with an arrow. Individuals with Roman numeric labels were analyzed in this study. Audiometric features of the subjects are displayed as audiograms (air conduction). The thresholds of the right and left ears are shown.

For many years $A T P 2 B 2$ has been described as a modifier of $C D H 23$ [49], and it has only recently been hypothesized that loss of function mutations in this gene cause autosomal dominant NSHL [50]. The identification of an additional ADNSHL family carrying a nonsense variant strengthens previous findings, confirming the pathogenic role of the ATP2B2 gene.

Another example is Family 32, an Italian family affected by NSHL (Figure 4B). WES revealed the presence of a novel heterozygous deletion in the HOMER2 gene (NM_199330.2) (i.e., c.592_597delACCACA, p.(Thr198_Thr199del)) segregating within the family in an autosomal dominant fashion. To our knowledge, this represents the third independent NSHL family carrying a variant in this gene [12,51], definitely confirming its relevant role in the etiopathogenesis of hearing loss.

Finally, WES proved to be extremely efficient for the molecular diagnosis of clinically evident SHL. In particular, all the Usher patients (i.e., patients displaying HL and retinitis pigmentosa) were molecularly characterized, identifying homozygous or compound heterozygous mutations in USH2A and MYO7A genes (Table 2). Among the two suspected Alport patients enrolled in the study, one was a carrier of a variant in COL4A3 ((NM_000091.5) c.3943C > T, p.(Pro1315Ser)) inherited from the affected father, while the second individual did not display any pathogenic mutation in all the genes known to be causative of such syndrome. Finally, a patient clinically diagnosed with Treacher-Collins syndrome carried a frameshift deletion in TCOF1 ((NM_000356) c.4131_4135del, p.(K1380Efs * 11)) (Table 2).

\section{Discussion}

The definition of the molecular basis of HHL has always being a challenge for clinicians and geneticists. The development and application of a multi-step integrated strategy based on (1) an accurate clinical evaluation; (2) GJB2/GJB6/MT-RNR1 screening; (3) MLPA; and (4) WES has proved to be a powerful approach for the molecular diagnosis of HHL patients.

Regarding NSHL, our data confirmed the relevant role of the GJB2 gene responsible for $20 \%$ of cases, and identified STRC as the second major player in the Italian population, being causative of $6 \%$ 
of all NSHL patients. In this light, the application of MLPA, or other techniques able to identify CNVs, is becoming a crucial test for NSHL patients. Interestingly, in agreement with literature data [17], STRC deletions have been identified in patients revealing mild-to-moderate hearing loss (Figure 5), thus, supporting a possible genotype-phenotype correlation between these audiometric features and STRC loss.

\section{Audiograms of STRC patients}
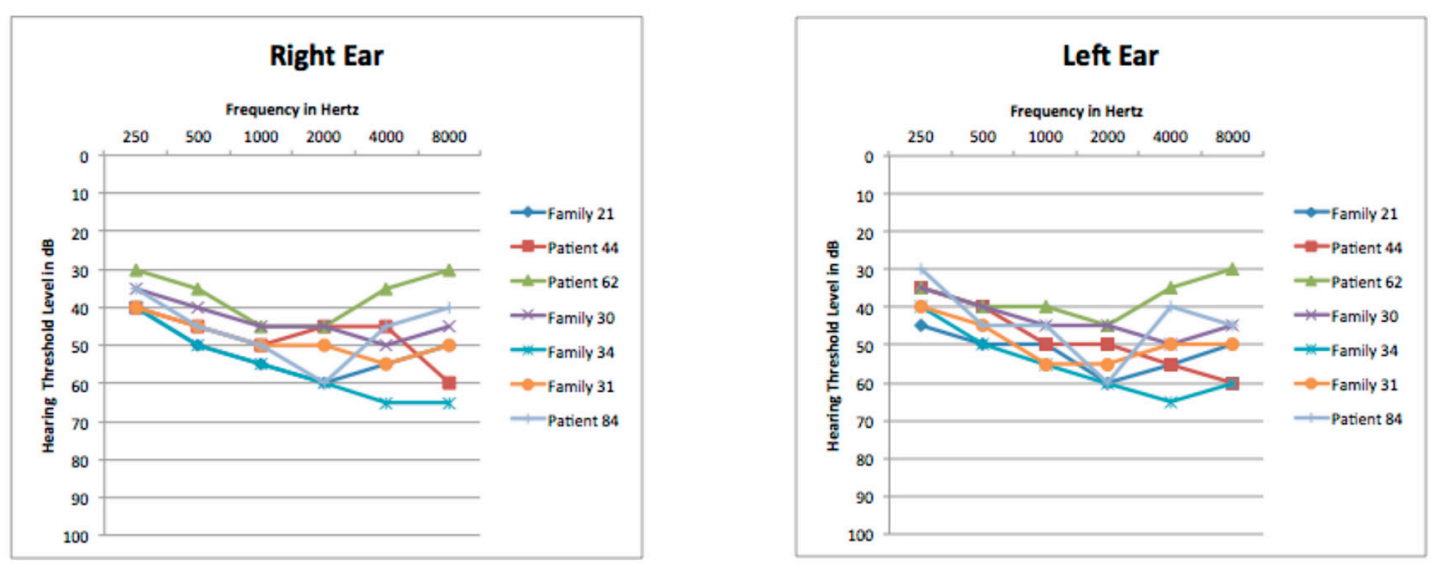

Figure 5. Audiometric features of the patients with STRC deletion/mutation. Audiograms of the patients with loss of function mutations or deletions of STRC gene display mild-to-moderate hearing loss. The thresholds of the right and left ears are shown.

On the other hand, no deletions in GJB6 have been detected, and the A1555G mitochondrial mutation. These results, combined with the outcomes of the previous works [10,52] suggest that GJB6 and MT-RNR1 are not a common cause of NSHL in the Italian population, despite their relevant role in other areas of the world.

WES allowed the identification of the genetic cause of HHL in $86 \%$ of the SHL patients and $23 \%$ of the NSHL subjects, revealing some unexpected findings in the latter case. Indeed, $4 \%$ of patients received a molecular diagnosis of syndromic HL, despite the first clinical evaluation in favor of NSHL, and multiple genetic causes of the clinical phenotype were identified in two families, hampering the interpretation of the sequencing data.

These findings emphasize the usefulness of WES compared to other approaches, such as the use of comprehensive gene panels. In fact, WES allowed at once (a) an early diagnosis of the syndromic cases that do not already show all the clinical signs or symptoms, (b) the possibility of unveiling unrelated co-existing genetic conditions, (c) the identification of new deafness candidate genes not previously described or only detected as private mutation/gene of a single family worldwide, thus resulting in a cost and time-saving approach.

The results of the present study highlight the complexity of HL and, more importantly, have obvious clinical outcomes. A correct molecular diagnosis provides patients with significant prognostic value and relevant heritability information and influences their management, leading to tailored medical surveillance and different therapeutic options. Moreover, in the case of HHL, it has been demonstrated that knowing the gene involved in the disease can help predict the response to cochlear implantation. As an example, patients carrying mutations in the GJB2 gene show an excellent response to cochlear implants. In contrast, those with mutations involving genes that affect the cochlear nerve itself gave worse post-implant performance [53]. Knowing this issue before implantation can help define expectations about post-implant auditory function. 
Overall, all the examples discussed above point out the complexity of HHL (both syndromic and non-syndromic). When dealing with this phenotype, it is essential to be aware of the difficulties encountered in choosing the most effective approach to arrive at a correct molecular diagnosis. With this in mind, the collaboration between geneticists, clinicians, and otolaryngologists, who have an in-depth knowledge of the clinical features of hearing loss and the genes involved, is fundamental to achieve the ultimate goal of unraveling the genetic bases of HHL and improving the lives of patients.

Author Contributions: Conceptualization, A.M. and G.G.; methodology, A.M., S.L., and G.P.; software, A.M., S.L., and G.G.; validation, S.L., and G.P.; formal analysis, A.M., F.F., and G.G.; investigation, B.S. and F.F.; resources, E.C., F.M.M., D.M., M.Z., C.G., U.A., and M.S.; data curation, A.M., and G.G.; writing—original draft preparation, A.M.; writing—review and editing, G.G.; visualization, A.M., S.L., and G.G.; supervision, F.F. and G.G.; project administration, A.M. and G.G.; funding acquisition: G.G. All authors have read and agreed to the published version of the manuscript.

Funding: This research was supported by D70-RESRICGIROTTO to GG, BENEFICENTIA Stiftung to G.G., and SG-2018-12367867 to A.M. The funders had no role in study design, data collection, and analysis, decision to publish, or preparation of the manuscript.

Acknowledgments: We gratefully acknowledge Martina Bradaschia for the English revision of the manuscript.

Conflicts of Interest: The authors declare no conflict of interest.

\section{References}

1. Allen, S.B.; Goldman, J. Hearing, Inner Ear, Syndromic Sensorineural Loss; StatPearls Publishing: Tampa, FL, USA, 2019.

2. Likar, T.; Hasanhodžić, M.; Teran, N.; Maver, A.; Peterlin, B.; Writzl, K. Diagnostic outcomes of exome sequencing in patients with syndromic or non-syndromic hearing loss. PLoS ONE 2018, 13. [CrossRef] [PubMed]

3. Pandey, S.; Pandey, M. Advances in Genetic Diagnosis and Treatment of Hearing Loss-A Thirst for Revolution. In Update On Hearing Loss; InTech: London, UK, 2015.

4. Smith, R.J.; Bale, J.F.; White, K.R. Sensorineural hearing loss in children. Lancet 2005, 365, 879-890. [CrossRef]

5. Korver, A.M.H.; Smith, R.J.H.; Van Camp, G.; Schleiss, M.R.; Bitner-Glindzicz, M.A.K.; Lustig, L.R.; Usami, S.I.; Boudewyns, A.N. Congenital hearing loss. Nat. Rev. Dis. Prim. 2017, 3, 16094. [CrossRef] [PubMed]

6. Ideura, M.; Nishio, S.Y.; Moteki, H.; Takumi, Y.; Miyagawa, M.; Sato, T.; Kobayashi, Y.; Ohyama, K.; Oda, K.; Matsui, T.; et al. Comprehensive analysis of syndromic hearing loss patients in Japan. Sci. Rep. $2019,9$. [CrossRef]

7. Wolfrum, U.; Nagel-Wolfrum, K. The Usher Syndrome, a Human Ciliopathy. Klin. Monbl. Augenheilkd. 2018, 235, 273-280. [CrossRef]

8. Ahmed, J.D.N.; Mui, R.K.; Masood, S. Waardenburg Syndrome. J. Med Genet. 2020, 34, 656-665.

9. Bademci, G.; Cengiz, F.B.; Foster, J.; Duman, D.; Sennaroglu, L.; Diaz-Horta, O.; Atik, T.; Kirazli, T.; Olgun, L.; Alper, H.; et al. Variations in Multiple Syndromic Deafness Genes Mimic Non-syndromic Hearing Loss. Sci. Rep. 2016, 6. [CrossRef]

10. Morgan, A.; Lenarduzzi, S.; Cappellani, S.; Pecile, V.; Morgutti, M.; Orzan, E.; Ghiselli, S.; Ambrosetti, U.; Brumat, M.; Gajendrarao, P.; et al. Genomic Studies in a Large Cohort of Hearing Impaired Italian Patients Revealed Several New Alleles, a Rare Case of Uniparental Disomy (UPD) and the Importance to Search for Copy Number Variations. Front. Genet. 2018, 9, 681. [CrossRef]

11. Cabanillas, R.; Diñeiro, M.; Cifuentes, G.A.; Castillo, D.; Pruneda, P.C.; Álvarez, R.; Sánchez-Durán, N.; Capín, R.; Plasencia, A.; Viejo-Díaz, M.; et al. Comprehensive genomic diagnosis of non-syndromic and syndromic hereditary hearing loss in Spanish patients. BMC Med. Genom. 2018, 11. [CrossRef]

12. Azaiez, H.; Decker, A.R.; Booth, K.T.; Simpson, A.C.; Shearer, A.E.; Huygen, P.L.M.; Bu, F.; Hildebrand, M.S.; Ranum, P.T.; Shibata, S.B.; et al. HOMER2, a stereociliary scaffolding protein, is essential for normal hearing in humans and mice. PLoS Genet. 2015, 11, e1005137. [CrossRef]

13. Morgan, A.; Koboldt, D.C.; Barrie, E.S.; Crist, E.R.; García García, G.; Mezzavilla, M.; Faletra, F.; Mihalic Mosher, T.; Wilson, R.K.; Blanchet, C.; et al. Mutations in PLS1, encoding fimbrin, cause autosomal dominant nonsyndromic hearing loss. Hum. Mutat. 2019. [CrossRef] [PubMed] 
14. Del Castillo, I.; Moreno-Pelayo, M.A.; Del Castillo, F.J.; Brownstein, Z.; Marlin, S.; Adina, Q.; Cockburn, D.J.; Pandya, A.; Siemering, K.R.; Chamberlin, G.P.; et al. Prevalence and Evolutionary Origins of the del(GJB6-D13S1830) Mutation in the DFNB1 Locus in Hearing-Impaired Subjects: A Multicenter Study. Am. J. Hum. Genet. 2003, 73, 1452-1458. [CrossRef]

15. Cama, E.; Melchionda, S.; Palladino, T.; Carella, M.; Santarelli, R.; Genovese, E.; Benettazzo, F.; Zelante, L.; Arslan, E. Hearing loss features in GJB2 biallelic mutations and GJB2/GJB6 digenic inheritance in a large Italian cohort. Int. J. Audiol. 2009, 48, 12-17. [CrossRef] [PubMed]

16. Morton, C.C.; Nance, W.E. Newborn Hearing Screening-A Silent Revolution. N. Engl. J. Med. 2006, 354, 2151-2164. [CrossRef]

17. Yokota, Y.; Moteki, H.; Nishio, S.Y.; Yamaguchi, T.; Wakui, K.; Kobayashi, Y.; Ohyama, K.; Miyazaki, H.; Matsuoka, R.; Abe, S.; et al. Frequency and clinical features of hearing loss caused by STRC deletions. Sci. Rep. 2019, 9. [CrossRef]

18. Clark, J.G. Uses and abuses of hearing loss classification. ASHA 1981, 23, 493-500.

19. Del Castillo, I.; Villamar, M.; Moreno-Pelayo, M.A.; del Castillo, F.J.; Alvarez, A.; Tellería, D.; Menéndez, I.; Moreno, F. A deletion involving the connexin 30 gene in nonsyndromic hearing impairment. N. Engl. J. Med. 2002, 346, 243-249. [CrossRef]

20. Danecek, P.; Auton, A.; Abecasis, G.; Albers, C.A.; Banks, E.; DePristo, M.A.; Handsaker, R.E.; Lunter, G.; Marth, G.T.; Sherry, S.T.; et al. The variant call format and VCFtools. Bioinformatics 2011, 27, 2156-2158. [CrossRef]

21. Wang, K.; Li, M.; Hakonarson, H. ANNOVAR: Functional annotation of genetic variants from high-throughput sequencing data. Nucleic Acids Res. 2010, 38, e164. [CrossRef]

22. Adzhubei, I.; Jordan, D.M.; Sunyaev, S.R. Predicting functional effect of human missense mutations using PolyPhen-2. Curr. Protoc. Hum. Genet. 2013, 7. [CrossRef]

23. Ng, P.C.; Henikoff, S. SIFT: Predicting amino acid changes that affect protein function. Nucleic Acids Res. 2003, 31, 3812-3814. [CrossRef] [PubMed]

24. Schwarz, J.M.; Rödelsperger, C.; Schuelke, M.; Seelow, D. MutationTaster evaluates disease-causing potential of sequence alterations. Nat. Methods 2010, 7, 575-576. [CrossRef] [PubMed]

25. Chun, S.; Fay, J.C. Identification of deleterious mutations within three human genomes. Genome Res. 2009, 19, 1553-1561. [CrossRef] [PubMed]

26. Kircher, M.; Witten, D.M.; Jain, P.; O’Roak, B.J.; Cooper, G.M.; Shendure, J. A general framework for estimating the relative pathogenicity of human genetic variants. Nat. Genet. 2014, 46, 310-315. [CrossRef] [PubMed]

27. Pollard, K.S.; Hubisz, M.J.; Rosenbloom, K.R.; Siepel, A. Detection of nonneutral substitution rates on mammalian phylogenies. Genome Res. 2010, 20, 110-121. [CrossRef] [PubMed]

28. Cooper, G.M.; Stone, E.A.; Asimenos, G.; NISC Comparative Sequencing Program; Green, E.D.; Batzoglou, S.; Sidow, A. Distribution and intensity of constraint in mammalian genomic sequence. Genome Res. 2005, 15, 901-913. [CrossRef] [PubMed]

29. Desmet, F.-O.; Hamroun, D.; Lalande, M.; Collod-Béroud, G.; Claustres, M.; Béroud, C. Human Splicing Finder: An online bioinformatics tool to predict splicing signals. Nucleic Acids Res. 2009, 37, e67. [CrossRef]

30. Shearer, A.E.; Kolbe, D.L.; Azaiez, H.; Sloan, C.M.; Frees, K.L.; Weaver, A.E.; Clark, E.T.; Nishimura, C.J.; Black-Ziegelbein, E.A.; Smith, R.J.H. Copy number variants are a common cause of non-syndromic hearing loss. Genome Med. 2014, 6, 37. [CrossRef]

31. McGuirt, W.T.; Prasad, S.D.; Griffith, A.J.; Kunst, H.P.M.; Green, G.E.; Shpargel, K.B.; Runge, C.; Huybrechts, C.; Mueller, R.F.; Lynch, E.; et al. Mutations in COL11A2 cause non-syndromic hearing loss (DFNA13). Nat. Genet. 1999, 23, 413-419. [CrossRef]

32. Vona, B.; Hofrichter, M.A.H.; Neuner, C.; Schröder, J.; Gehrig, A.; Hennermann, J.B.; Kraus, F.; Shehata-Dieler, W.; Klopocki, E.; Nanda, I.; et al. DFNB16 is a frequent cause of congenital hearing impairment: Implementation of STRC mutation analysis in routine diagnostics. Clin. Genet. 2015, 87, 49-55. [CrossRef]

33. Numakura, C.; Lin, C.; Ikegami, T.; Guldberg, P.; Hayasaka, K. Molecular analysis in Japanese patients with Charcot-Marie-Tooth disease: DGGE analysis for PMP22, MPZ, and Cx32/GJB1 mutations. Hum. Mutat. 2002, 20, 392-398. [CrossRef] [PubMed] 
34. Wattenhofer, M.; Reymond, A.; Falciola, V.; Charollais, A.; Caille, D.; Borel, C.; Lyle, R.; Estivill, X.; Petersen, M.B.; Meda, P.; et al. Different mechanisms preclude mutant CLDN14 proteins from forming tight junctions in vitro. Hum. Mutat. 2005, 25, 543-549. [CrossRef] [PubMed]

35. Ruf, R.G.; Xu, P.X.; Silvius, D.; Otto, E.A.; Beekmann, F.; Muerb, U.T.; Kumar, S.; Neuhaus, T.J.; Kemper, M.J.; Raymond, R.M.; et al. SIX1 mutations cause branchio-oto-renal syndrome by disruption of EYA1-SIX1-DNA complexes. Proc. Natl. Acad. Sci. USA 2004, 101, 8090-8095. [CrossRef] [PubMed]

36. Sheppard, S.; Biswas, S.; Li, M.H.; Jayaraman, V.; Slack, I.; Romasko, E.J.; Sasson, A.; Brunton, J.; Rajagopalan, R.; Sarmady, M.; et al. Utility and limitations of exome sequencing as a genetic diagnostic tool for children with hearing loss. Genet. Med. 2018, 20, 1663-1676. [CrossRef]

37. Lenarduzzi, S.; Morgan, A.; Faletra, F.; Cappellani, S.; Morgutti, M.; Mezzavilla, M.; Peruzzi, A.; Ghiselli, S.; Ambrosetti, U.; Graziano, C.; et al. Next generation sequencing study in a cohort of Italian patients with syndromic hearing loss. Hear. Res. 2019, 381. [CrossRef]

38. Rodríguez-Ballesteros, M.; Reynoso, R.; Olarte, M.; Villamar, M.; Morera, C.; Santarelli, R.; Arslan, E.; Medá, C.; Curet, C.; Völter, C.; et al. A multicenter study on the prevalence and spectrum of mutations in the otoferlin gene ( OTOF) in subjects with nonsyndromic hearing impairment and auditory neuropathy. Hum. Mutat. 2008, 29, 823-831. [CrossRef]

39. Magliulo, G.; Iannella, G.; Gagliardi, S.; Iozzo, N.; Plateroti, R.; Mariottini, A.; Torricelli, F. Usher's Syndrome Type II: A Comparative Study of Genetic Mutations and Vestibular System Evaluation. Otolaryngol. Head Neck Surg. 2017, 157, 853-860. [CrossRef]

40. Rivolta, C.; Sweklo, E.A.; Berson, E.L.; Dryja, T.P. Missense mutation in the USH2A gene: Association with recessive retinitis pigmentosa without hearing loss. Am. J. Hum. Genet. 2000, 66, 1975-1978. [CrossRef]

41. Van Wijk, E.; Pennings, R.J.E.; Te Brinke, H.; Claassen, A.; Yntema, H.G.; Hoefsloot, L.H.; Cremers, F.P.M.; Cremers, W.R.J.; Kremer, H. Identification of 51 Novel Exons of the Usher Syndrome Type 2A (USH2A) Gene That Encode Multiple Conserved Functional Domains and That Are Mutated in Patients with Usher Syndrome Type II. Am. J. Hum. Genet. 2004, 74, 738-744. [CrossRef]

42. Choi, B.Y.; Park, G.; Gim, J.; Kim, A.R.; Kim, B.J.; Kim, H.S.; Park, J.H.; Park, T.; Oh, S.H.; Han, K.H.; et al. Diagnostic Application of Targeted Resequencing for Familial Nonsyndromic Hearing Loss. PLoS ONE 2013, 8. [CrossRef]

43. Ebermann, I.; Phillips, J.B.; Liebau, M.C.; Koenekoop, R.K.; Schermer, B.; Lopez, I.; Schäfer, E.; Roux, A.F.; Dafinger, C.; Bernd, A.; et al. PDZD7 is a modifier of retinal disease and a contributor to digenic Usher syndrome. J. Clin. Invest. 2010, 120, 1812-1823. [CrossRef]

44. Shearer, A.E.; DeLuca, A.P.; Hildebrand, M.S.; Taylor, K.R.; Gurrola, J.; Scherer, S.; Scheetz, T.E.; Smith, R.J.H. Comprehensive genetic testing for hereditary hearing loss using massively parallel sequencing. Proc. Natl. Acad. Sci. USA 2010, 107, 21104-21109. [CrossRef]

45. Sloan-Heggen, C.M.; Bierer, A.O.; Shearer, A.E.; Kolbe, D.L.; Nishimura, C.J.; Frees, K.L.; Ephraim, S.S.; Shibata, S.B.; Booth, K.T.; Campbell, C.A.; et al. Comprehensive genetic testing in the clinical evaluation of 1119 patients with hearing loss. Hum. Genet. 2016, 135, 441-450. [CrossRef] [PubMed]

46. Atik, T.; Onay, H.; Aykut, A.; Bademci, G.; Kirazli, T.; Tekin, M.; Ozkinay, F. Comprehensive analysis of deafness genes in families with autosomal recessive nonsyndromic hearing loss. PLoS ONE 2015, 10. [CrossRef]

47. Tassabehji, M.; Read, A.P.; Newton, V.E.; Patton, M.; Gruss, P.; Harris, R.; Strachan, T. Mutations in the PAX3 gene causing Waardenburg syndrome type 1 and type 2. Nat. Genet. 1993, 3, 26-30. [CrossRef] [PubMed]

48. Chen, M.H.; Walsh, C.A. FLNA-Related Periventricular Nodular Heterotopia; University of Washington: Seattle, WA, USA, 1993.

49. Schultz, J.M.; Yang, Y.; Caride, A.J.; Filoteo, A.G.; Penheiter, A.R.; Lagziel, A.; Morell, R.J.; Mohiddin, S.A.; Fananapazir, L.; Madeo, A.C.; et al. Modification of human hearing loss by plasma-membrane calcium pump PMCA2. N. Engl. J. Med. 2005, 352, 1557-1564. [CrossRef] [PubMed]

50. Smits, J.J.; Oostrik, J.; Beynon, A.J.; Kant, S.G.; de Koning Gans, P.A.M.; Rotteveel, L.J.C.; Klein Wassink-Ruiter, J.S.; Free, R.H.; Maas, S.M.; van de Kamp, J.; et al. De novo and inherited loss-of-function variants of ATP2B2 are associated with rapidly progressive hearing impairment. Hum. Genet. 2019, 138, 61-72. [CrossRef] [PubMed] 
51. Lu, X.; Wang, Q.; Gu, H.; Zhang, X.; Qi, Y.; Liu, Y. Whole exome sequencing identified a second pathogenic variant in HOMER2 for autosomal dominant non-syndromic deafness. Clin. Genet. 2018, 94, 419-428. [CrossRef]

52. Vozzi, D.; Morgan, A.; Vuckovic, D.; D’Eustacchio, A.; Abdulhadi, K.; Rubinato, E.; Badii, R.; Gasparini, P.; Girotto, G. Hereditary hearing loss: A 96 gene targeted sequencing protocol reveals novel alleles in a series of Italian and Qatari patients. Gene 2014, 542, 209-216. [CrossRef]

53. Shearer, A.E.; Eppsteiner, R.W.; Frees, K.; Tejani, V.; Sloan-Heggen, C.M.; Brown, C.; Abbas, P.; Dunn, C.; Hansen, M.R.; Gantz, B.J.; et al. Genetic variants in the peripheral auditory system significantly affect adult cochlear implant performance. Hear. Res. 2017, 348, 138-142. [CrossRef]

Publisher's Note: MDPI stays neutral with regard to jurisdictional claims in published maps and institutional affiliations.

(C) 2020 by the authors. Licensee MDPI, Basel, Switzerland. This article is an open access article distributed under the terms and conditions of the Creative Commons Attribution (CC BY) license (http://creativecommons.org/licenses/by/4.0/). 